\title{
A NON-INVASIVE GLUCOSE ANALYSIS MODEL WITH A CARBON NANOTUBE SENSOR
}

\section{Nicolescu Adrian Eracle',Rusali Lavinia1,Vasile Monica1}

${ }^{1}$ Faculty of Medicine, University "Ovidius" of Constanța, Romania

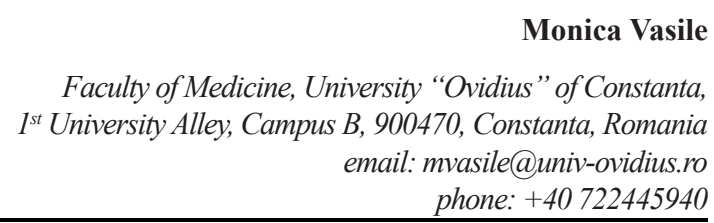

ABSTRACT

The development and studies related to carbon nanotubes, due to their physical properties and small sizes, are revealing new applications in the medical field. The present paper is proposing a non-invasive method of patient glycemia measurement by mean of carbon nanotubes based sensor. Due to their small sizes and elastic properties, carbon nanotubes are able to oscillate under the weight of small molecules capable of fixing on their body structure. After piezoelectric activation, the carbon nanotubes sensor based, is able to detect the variation of oscillations produced in nanotubes due to acetone molecules diffused through a selectively permeable membrane from the exhaled air, and attached to the nanotubes. The mathematical model taken into accont shows a linear dependence between the concentration of acetone in blood and glycemia, and makes the carbon nanotube sensor suitable for indirect estimation of the glycemia by noninvasive measurements made on the exhaled air sample, providing zero risk for the patient and absence of stress during the measurements.

Keywords: carbon nanotubes; sensor; blood sugar; harmonic linear oscillator; non-invasive method.

\section{Introduction}

The acetone concentration in the breath of diabetic patients is much higher than in a healthy person. Studies $(1,2)$ have shown the proportionality between the acetone concentration in the exhaled air and the acetone concentration in the blood and, respectively, the linear dependence between the acetone concentration in the blood and blood sugar.

The analysis model proposed in this article incorporates the detection of acetone from the patient's breath using a carbon nanotube sensor. In this sensor, each of the carbon nanotubes is fixed at one end of a substrate, and at the other end it is free. Carbon nanotubes are placed on the surface of a selective membrane. When a patient's breathing sample is introduced, the membrane retains all other molecules, allowing only acetone molecules to pass. Piezoelectric activation is used to put the nanotubes in a state of oscillation, thus the oscillation frequencies of the nanotubes are measured and their spectrum is drawn.

If an acetone molecule is attached to a carbon nanotube, then its mass distribution changes, and, by default, the frequency of oscillations changes. The new oscillation frequencies of the nanotubes are measured and the new oscillation spectrum is plotted.

By measuring the relative frequency variation following coupling of acetone 
molecules, detection can be made up to the level of a single acetone molecule and, implicitly, can be measured indirectly.

\section{Materials and methods}

The mathematical model for the carbon nanotube used as a sensor for acetone

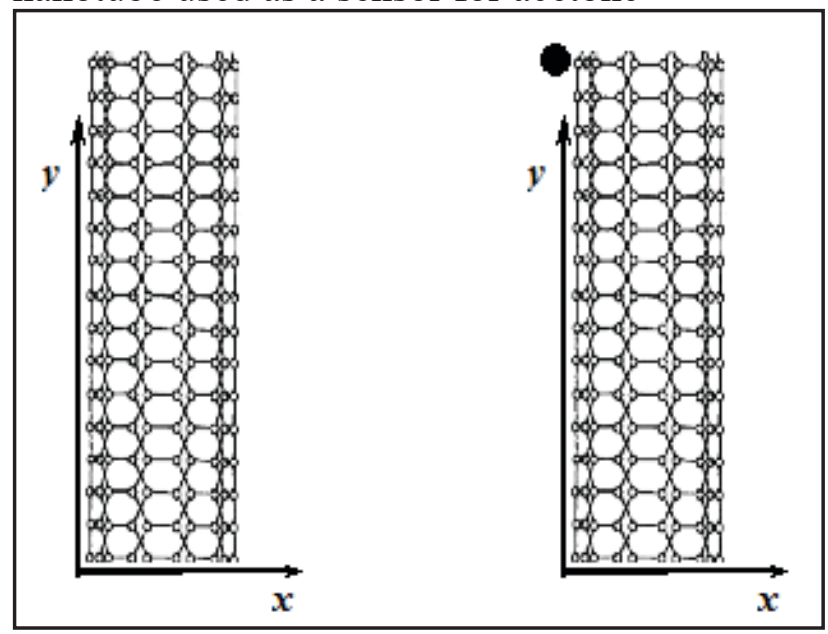

Figure 1. Carbon nanotube before and after capture of an acetone molecule.

For the carbon nanotube oscillator we will use the model of the homogeneous elastic bar fixed at one end and the other free end. By activating piezoelectrically, the carbon nanotube will freely oscillate in the y direction.

In the approximation of small oscillations, the oscillation of the nanotube can be expressed by equation (3):

$$
E I \frac{\partial^{2} y}{\partial x^{2}}+\rho A \frac{\partial^{2} y}{\partial t^{2}}=0
$$

where E is Young's modulus of the carbon nanotube, I is the moment of inertia of the nanotube, $\rho$ is the density of the carbon nanotube, $\mathrm{A}$ is the cross-section area of the carbon nanotube at the distance $\mathrm{x}$ from $\mathrm{O}$, and $\mathrm{y}$ is the elongation of the nanotube at $\mathrm{x}$.

The carbon nanotube (tube with uniformly distributed mass) can be modeled as a harmonic (spring-body) linear oscillator, the body being located at distance L from the substrate which harmonically oscillates similarly to the free end of the nanotube(4).

The proper frequency of the oscillator equivalent to the carbon nanotube, in the case of small oscillations can be expressed by the relation:

$$
v=\frac{1}{2 \pi} \sqrt{\frac{k}{m_{e q}}}
$$

where $\mathrm{k}$ is the elasticity constant of the equivalent spring, and $\mathrm{m}_{\mathrm{eq}}$ is the equivalent mass attached to the end of the spring. An additional mass attached to the end of the carbon nanotube will cause modifications in the frequency of its small oscillations.

\section{Results and discussion}

Determining the elasticity constant corresponding to the spring

We consider a carbon nanotube of length $\mathrm{L}$ that bends under the action of force $F$ at its end (5). We analyse the static case considering that:

$$
\begin{gathered}
y(0)=0(3) \\
\frac{d y}{d x}(0)=0(4) \\
\frac{d^{2} y}{d x^{2}}=\frac{F(L-x)}{E I}(5)
\end{gathered}
$$

From the expression of equation (5) of the bending elastic beam, it turns out that $y$ (elongation) must be a polimomial function of $3^{\text {th }}$ order in $\mathrm{x}$

$$
y(x)=A+B x+C x^{2}+D x^{3}
$$

From (3), (4), (5) şi (6), the result is that:

$$
\begin{gathered}
A=B=0(7) \\
C=\frac{F}{2 E I}(8) \\
D=-\frac{F}{6 E I}(9)
\end{gathered}
$$

If we replace (7), (8), (9) in (6), it turns out that the equation of the shape of the nanotube approximated with an elastic beam has the expression:

$$
y(x)=\frac{F x^{2}(3 L-x)}{6 E I}
$$

The maximum deformation is obtained from (10) for $\mathrm{x}=\mathrm{L}$ :

$$
y_{\max }=y(L)=\frac{F L^{3}}{3 E I}
$$

The elasticity constant of the spring equivalent to the nanotube is 


$$
k=\frac{F}{y(L)}=\frac{3 E I}{L^{3}}
$$

Determination of the mass of the oscillator equivalent to the carbon nanotube

We consider the system formed of the nanotube shaped as a beam and the acetone molecule, set in motion by oscillation. The total kinetic energy of the system can be expressed as the sum of the kinetic energy of the carbon nanotube and that of the mass attached to its free end:

$$
E=E_{C N T}+E_{\text {acetona }}(13)
$$

For the equivalent oscillator, kinetic energy can be expressed by the relation

$$
E=\frac{1}{2} m_{e q} \omega^{2} \cdot y^{2}(L)
$$

Considering $\rho$ is the density of the nanotube, $\mathrm{A}$ is the area of the cross-section and $\mathrm{L}$ its length, we can express the mass of an infinitesimal element of thickness $\mathrm{dx}$ from the carbon nanotube

$$
d m_{C N T}=\rho A d x
$$

The kinetic energy of the infinitesimal element of the nanotube can be expressed by

$$
d E_{C N T}=\frac{1}{2} \omega^{2} y^{2} d m_{C N T}=\frac{1}{2} \rho A \omega^{2} y^{2} d x
$$

And the kinetic energy of the nanotube, according to (16) is

$$
E_{C N T}=\frac{1}{2} \int_{0}^{L} \rho A \omega^{2} y^{2} d x
$$

Taking into account equation (10), it follows that (17) can be expressed as

$$
E_{C N T}=\frac{1}{2} \int_{0}^{L} \rho A \omega^{2}\left[\frac{F x^{2}(3 L-x)^{2}}{6 E I}\right] d x=\frac{1}{2} \rho A \omega^{2}\left(\frac{33 L}{140}\right)\left(\frac{F L^{3}}{3 E I}\right)^{2}
$$

The kinetic energy of oscillation of mass $\mathrm{m}$ of acetone is

$$
E_{\text {acetona }}=\frac{1}{2} m \omega^{2} y^{2}(L)=\frac{1}{2} m \omega^{2}\left(\frac{F L^{3}}{3 E I}\right)^{2}
$$

If we replace (18) and (19) with (13) the result is

$$
E=\frac{1}{2} \omega^{2}\left(\frac{33 L}{140} \rho A+m\right)\left(\frac{F L^{3}}{3 E I}\right)^{2}=\frac{1}{2} \omega^{2}\left(\frac{33 L}{140} \rho A+m\right) \cdot y^{2}(L)
$$

If we compare (14) with (20), it follows that an oscillating nanotube can be modeled as a spring of elasticity constant $\mathrm{k}$ and an equivalent mass body at its end given by the expression

$$
m_{e q}=\frac{33 L}{140} \rho A+m
$$

Using the simplified body-spring model, the frequency of the carbon nanotube with a mass $m$ of acetone at its end can be calculated by

$$
v=\frac{1}{2 \pi} \sqrt{\frac{k}{m_{e q}}}=\frac{1}{2 \pi} \sqrt{\frac{\frac{3 E I}{L^{3}}}{\frac{33 L}{140} \rho A+m}}
$$

If no acetone molecule is attached, then the oscillation frequency of the nanotube is:

$$
v_{0}=\frac{1}{2 \pi} \sqrt{\frac{140 E I}{11 \rho A L^{4}}}
$$

The result from (22) and (23) is:

$$
v=v_{0} \frac{1}{\sqrt{1+\frac{140 m}{11 \rho A L}}}
$$

Relative frequency variation:

$$
\frac{\Delta v}{v_{0}}=1-\frac{1}{\sqrt{1+\frac{140 m}{11 \rho A L}}}
$$

The mass of the acetone attached to the nanotube derives from (25) :

$$
m=\frac{33 \rho A L}{140}\left[\frac{1}{\left(1-\frac{\Delta v}{v_{0}}\right)^{2}}-1\right]
$$

We evaluate the relative variation of the oscillation frequency after coupling the acetone molecules and calculate the mass of acetone and implicitly its concentration in the patient's breathing.

\section{Results}

The concentration of acetone in the blood can be expressed according to the concentration of acetone in the exhaled air based on the relation (6):

$$
C_{b}=C_{a} / \Lambda
$$

where $C_{a}$ is the acetone concentration in the 
air (ppmv), $C_{b}$ este is the acetone concentration in the blood $(\mu \mathrm{g} / \ell)$, and $\Lambda=12,825 \ell / \mu \mathrm{g}$ is an empirical constant, dependent on the molar mass of acetone and its molar volume at temperature of $310 \mathrm{~K}$.

Between the blood glucose and the concentration of acetone in the exhaled air there is an empirical formula (7)

$$
B G=\alpha C_{a}+\beta
$$

obtained from the interpretation of the results of the study, where BG is expressed in $\frac{m g}{d \ell}, C_{a}$ is the concentration of acetone in air expressed in ppmv, and the constants (8) $\alpha=91.38 \frac{m g}{d \ell \cdot p p m v}$, and $\beta=6,3743 \frac{\mathrm{mg}}{\mathrm{d} \ell}$.

Using equations (26), (27) and (28), the conversion of mechanical size (frequency of carbon nanotube oscillation) is obtained in medical analysis (blood glucose). Figure 2 shows the block diagram of the described sensor.

\section{Bonclusions}

The physical properties of nanotubes make them suitable for different aplications in medical area. Patients with diabetes are already submitted to a lot of stress due to the repeted, invasive, daily glycemic tests. Raseing the stress to the patient is inconvinient, knowing that physical stress factor is contributing in a negative manner to their medical issue. The use of carbon nanotube based sensors, due to their small size and physical properties make them very appropriate for noninvaseing measurements, with fast response and good precision, with reduced stress to the individual, in monitoring a vital parameter for patients with diabities.

\section{Acknowledgment}

The first author is supported by the project ANTREPRENORDOC, in the framework of
Human Resources Development Operational Programme 2014-2020, financed from the European Social Fund under the contract number 36355/23.05.2019 HRD OP /380/6/13 - SMIS Code: 123847.

\section{References}

1. Jones AW. Measuring and reporting the concentration of acetaldehyde in human breath. Alcohol Alcohol. 1995 May;30(3):271-85

2. Brechner VL, Bethune RW. Determination of acetone concentration in arterial blood by vapor phase chromatography of alveolar gas. Diabetes. 1965 Oct;14(10):663-5.

3. Chowdhury R, Adhikari S, Mitchell J. Vibrating carbon nanotube based biosensors. Physica E: Low-dimensional Systems and Nanostructures. 2009 2009/12/01/;42(2):104-9.

4. Hierold C, Jungen A, Stampfer C, Helbling T. Nano electromechanical sensors based on carbon nanotubes. Sensors and Actuators A: Physical. 2007 2007/05/01/;136(1):51-61.

5. He JH, Sun S-q, Ye J-S, Lim TM. SelfAssembly Carbon Nanotubes on Cantilever Biosensor for Sensitivity Enhancement. Journal of Physics: Conference Series. 2006 2006/04/01;34:423-8.

6. Anderson JC. Measuring breath acetone for monitoring fat loss: Review. Obesity (Silver Spring). 2015 Dec;23(12):2327-34.

7. Yan K, Zhang D. Blood glucose prediction by breath analysis system with feature selection and model fusion. 2014 36th Annual International Conference of the IEEE Engineering in Medicine and Biology Society. 2014:6406-9.

8. Hassan T, Rehman T, Aziz QA, Salman A. Blood Glucose Level Measurement from Breath Analysis. International Journal of Biomedical and Biological Engineering. 2018;12(9):379-82

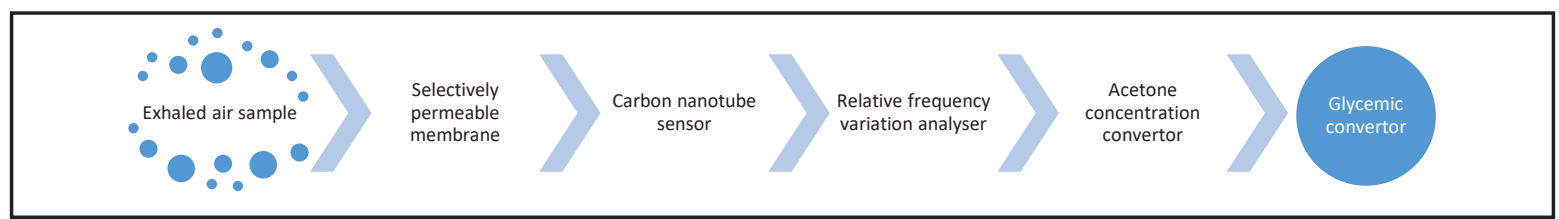

Figure 2. Block diagram of the experimental device. 